\title{
The Remarkable Binary Pulsar PSR B1259-63
}

\author{
Simon Johnston \\ Research Centre for Theoretical Astrophysics, Univ. Sydney, Australia
}

\begin{abstract}
PSR B1259-63 is a unique system consisting of a young radio pulsar in orbit about a $10 \mathrm{M}_{\odot}$ Be star. Its orbital period is 3.4 $\mathrm{yr}$ and the eccentricity close to 0.9 . Around periastron, the system is a radio and X-ray transient and shows changes in flux density, dispersion measure, rotation measure and scattering parameters on very short timescales. Timing observations show the equatorial plane of the Be star and the plane of the orbit are not aligned and the orbit precesses as a result. I briefly summarise 10 years of radio observations of PSR B125963 and the implications of these observations. Future work includes the determination of the orbital size through VLBI and observations at $\mathrm{TeV}$ energies.
\end{abstract}

\section{Introduction}

PSR B1259-63 was discovered in late 1989 as part of a galactic plane survey for pulsars (Johnston et al. 1992a). It was subsequently found to be in a long period, highly eccentric orbit about a $10 \mathrm{M}_{\odot}$ Be star (Johnston et al. 1992b). Be stars are characterised by a disk of material circling the equator of the star possibly thrown off by very fast rotation. The optical disk in this star extends to at least $20 \mathrm{R}_{*}$ (Johnston et al. 1994) which is comparable to the closest approach of the pulsar. The disk material is extremely dense near the stellar surface but falls off rapidly as a function of distance (perhaps as steep as $\mathbf{r}^{-4}$ ). The polar wind, by contrast is $\sim 10^{4}$ times less dense than the disk material and has an $\mathrm{r}^{-2}$ density profile. The distance to the system based on optical observations is about $1.5 \mathrm{kpc}$ (Johnston et al. 1994), considerably less than the $4.8 \mathrm{kpc}$ distance derived from the pulsar's dispersion measure.

The system has been extensively observed at high energies. This includes detection of the system at apastron by ROSAT (Cominsky et al. 1994), ASCA and OSSE (Hirayama et al. 1999) and detection at periastron by ASCA (Kaspi et al. 1995; Hirayama et al. 1996) and OSSE (Grove et al. 1995). The luminosity differs by about a factor of 20 from periastron to apastron and there is no evidence for any pulsed X-ray emission. Hirayama et al. (1999) has nicely summarised the high energy observations. Tavani \& Arons (1997) proposed a model where the X-ray emission is produced by shock emission in the interface between the pulsar wind and the stellar wind.

In this review I concentrate on the radio observations of the system and the implications of these observations. Virtually all this information has been 
published in refereed journals and interested readers should consult the original sources.

\section{Pulse Timing}

Timing of PSR B1259-63 from the discovery in 1990 to the end of 1997 was discussed in Wex et al. (1998). There are two main difficulties involved in the timing of this pulsar. The first is that the pulsar is relatively young and so suffers from timing noise which must be taken into account. Secondly, the pulsar is eclipsed for virtually 180 degrees of orbital angle and the lack of crucial timing information near periastron itself adds significantly to the error bars of the orbital elements. Our original timing model in which we included the effects of propellor-torque spindown (Manchester et al. 1995) has subsequently been shown to be incorrect.

The best fit model obtained by Wex et al. (1998) includes a $\ddot{P}$ term to account for the long-term behaviour of the timing noise, and $\dot{\omega}$ and $\dot{x}$, which both are understood to result from a precession of the orbit. This orbital precession can be explained by the classical spin-orbit coupling caused by the quadrupolar nature of the main-sequence star companion. The corresponding advance of periastron is negative and thus the companion should be tilted by more than 30 degrees with respect to the orbital plane. This can be explained by a (large) birth kick for the pulsar. We also showed that both tidal dissipation and frictional drag in the circumstellar matter is negligible for the future evolution of this system.

Subsequent timing observations have shown that the pulsar underwent a small glitch in 1998 (Wex, private communication). Timing continues at Parkes and we are looking forward to the next periastron in October 2000.

\section{Scintillation Properties}

The scintillation properties of PSR B1259-63 were discussed extensively in McClure-Griffiths et al. (1998). In that paper we determined the scintillation parameters of the pulsar as a function of orbital phase and concluded that there was no change in these parameters except during a small portion of the orbit around the time of periastron. The scintillation bandwidth is much less than expected if the scintillation were solely due to the background ISM. We demonstrated that the electron densities are not sufficiently large in either the wind of the Be star far from periastron or a putative Strömgren sphere to produce the observed scattering. The Sagittarius-Carina spiral arm is therefore the most likely origin of the scintillation far from periastron. At periastron, however, the electron density in the Be star disk is sufficiently large to counterbalance the 'lever-arm effect' of having the screen so close to the pulsar. This produces extremely strong scattering, and the resultant broadening of the pulsar image is sufficient to quench further scintillation in the ISM. 


\section{Pulsed Emission around Periastron}

We carried out extensive multifrequency observations of PSR B1259-63 during the periastron passage of 1994 and 1997 when the pulsar passed through the circumstellar disc of the Be star. In summary, and with the epoch of periastron (in days) denoted as $\mathcal{T}$, these observations show that

- the pulses became depolarized at $1.5 \mathrm{GHz}$ as early as $\mathcal{T}-94$ and at higher frequencies by $\mathcal{T}-30$.

- the dispersion measure (DM) increased steadily prior to periastron, reaching a value $11 \mathrm{~cm}^{-3}$ pc greater than the nominal DM during the last detection of the pulses before periastron on $\mathcal{T}-19$

- substantial scatter-broadening of the pulses at $1.5 \mathrm{GHz}$ was seen on $\mathcal{T}-30$, and that the pulsed flux density was also low on this date at $1.5 \mathrm{GHz}$

- scatter-broadening of the pulses was large and increased over a short timescale during observations made at $4.8 \mathrm{GHz}$ on $\mathcal{T}-19$

- no pulsed flux was detected between $\mathcal{T}-19$ and $\mathcal{T}+14$ inclusive

- the pulsar was re-detected post-periastron when there was little evidence for enhancement of DM or scatter-broadening, even at $1.5 \mathrm{GHz}$

- the rotation measure (RM) on $\mathcal{T}+24$, as measured at $8.4 \mathrm{GHz}$, was -6800 $\mathrm{rad} \mathrm{m}^{-2}$

- the magnetic field in the disc at about $45 R_{*}$ is $\sim 40 \mathrm{mG}$; this is the first direct measurement of the magnetic field in a Be star's disc

- the RM and percentage linear polarization changed on short time-scales (few minutes) during observations around $\mathcal{T}+30$

- two months after periastron the pulsar was still only partially polarized at $1.5 \mathrm{GHz}$

- three months after periastron, polarization had returned to normal levels.

Melatos et al. (1995) concluded from the observations of the 1994 periastron passage that the pulsar wind cannot dominate the Be star wind and that, in any case, the stellar wind cannot alone account for the changes in DM and RM observed. The stellar disk, however, can account for these changes and Melatos et al. showed that a thick disk with an exponential density profile and reasonable parameters for initial electron density and magnetic field could model the observations reasonably well. We also showed that the disk must be tilted relative to the orbital plane, a result which was later shown to be correct from the timing data (Wex et al. 1998). 


\section{Unpulsed Emission around Periastron}

In 1994 , we discovered that the system was a radio transient in that excess radio emission (i.e. in excess of the pulsar flux) was seen in over a period of 100 days before and after periastron. In 1997 we had an extensive monitoring campaign with the Australia Telescope Compact Array and we observed the source on 34 occasions from 128 days before periastron until 50 days after. The observations were at $1.4,2.4,4.8$ and $8.4 \mathrm{GHz}$ and employed pulsar binning such that we could easily separate on-pulse from off-pulse emission. We also observed with the Molonglo Observatory Synthesis Telescope at $843 \mathrm{MHz}$ on 15 occasions. The observations and results are described in detail in Johnston et al. (1999).

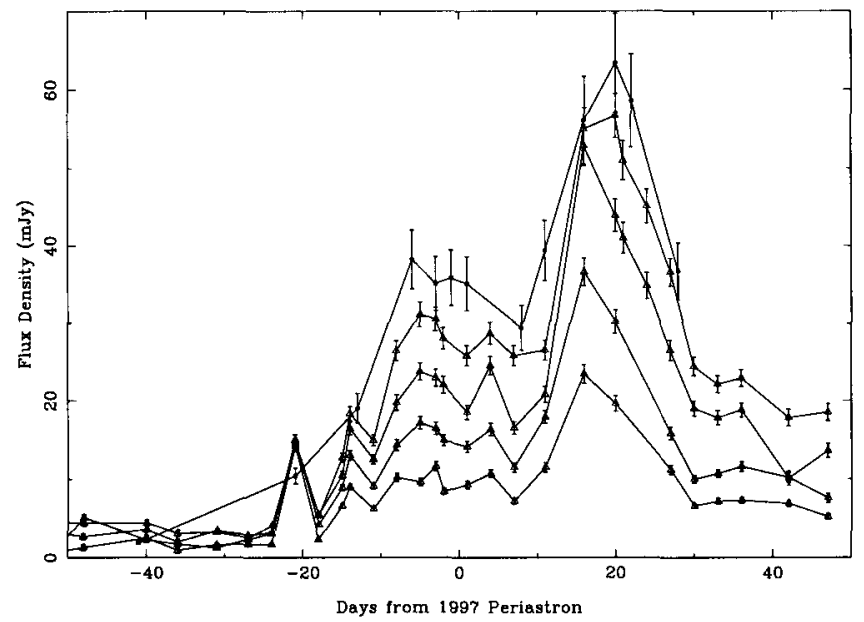

Figure 1. Flux densities of the PSR B1259-63 system around the 1997 periastron. The five curves, from top to bottom, represent the flux densities at $0.843,1.4,2.4,4.8$ and $8.4 \mathrm{GHz}$ repsectively. The pulsar flux density is only about $3 \mathrm{mJy}$ at these frequencies.

In brief, the observations reveal a relatively strong and rapidly varying but unpulsed radio source which persists from 22 days prior to periastron until 100 days after periastron. There are two peaks in the light curve near $\mathcal{T}-10$ and $\mathcal{T}+20$ (see Figure 1). For most of this time, the spectral index is $\sim-0.5$, consistent with optically thin synchrotron emission. We associate the peaks of the emission with relativistic electrons generated in the encounter of the pulsar wind with the equatorial disc of the Be star. The pulsar encounters the disk near $\mathcal{T}-10$ (consistent with the picture derived from timing and pulsed observations) and a synchrotron bubble forms within this high density region. The pulsar then moves on behind the disk leaving the synchrotron bubble to evolve. The pulsar re-enters the disk at $\mathcal{T}+20$ causing the formation of a second synchrotron bubble which evolves in its own right. A model involving adiabatic expansion of the emission region and variations in the fraction of the emission absorbed by the stellar disk gives a qualitative explanation of the observed radio flux density and spectral changes. There is a close relationship between the radio 
and $\mathrm{X}$-ray emission from the system, although they are probably generated by different populations of relativistic electrons. Detailed calculations on the emission mechanism and a more complete investigation of the implications for the parameters of the PSR B1259-63 system are given in Ball et al. (1999).

\section{Future Work - Periastron 2000}

Currently, high resolution VLBI observations are underway to determine the proper motion of the system and perhaps to resolve the pulsar orbit. The large inclination angle between the equatorial plane of the Be star and the orbital plane implies that the pulsar must have received a large kick at birth. In this case the proper motion is expected to be around $100 \mathrm{~km} / \mathrm{s}$ or so. Also, the pulsar orbit is of order a few milli-arcseconds (assuming a distance of $1.5 \mathrm{kpc}$ ) and it may be possible to resolve the orbit and hence determine the inclination angle of the system in the plane of the sky.

The UV photons from the Be star can be scattered to TeV energies during the interaction with the relativistic electrons from both the shocked and the unshocked pulsar wind (Kirk et al. 1999; Ball \& Kirk 1999). These models suggest that the system should be visible at $\mathrm{TeV}$ energies. The intensity of the $\mathrm{TeV}$ photons is a function of orbital phase and is expected to peak near periastron. The CANGAROO II telescope, located in South Australia, will observe PSR B1259-63 in mid-2000; it should have sufficient sensitivity to detect $\mathrm{TeV}$ photons from the system if the models are correct.

Further observations are also planned in the radio band. In particular, for the unpulsed emission, an investigation of how the flux decays beyond $\mathcal{T}+50$ is important. Also, the 'splashing' effect of the pulsar entering the disk (seen near $\mathcal{T}-21$ in Figure 1) needs to be investigated in more detail. Timing observations continue on a monthly basis and observations through the 2000 periastron will help determine the orbital parameters.

\section{Acknowledgements}

I would like to thank the SOC of IAU 177 for the opportunity to present this work at the Bonn meeting and Don Melrose for his continuing support of this project. There have been numerous collaborators on the 1259 project and I thank the following people in the hope that no-one is missing: Lewis Ball, Matthew Bailes, Matthew Britton, Duncan Campbell-Wilson, Andrew Lyne, J-P Macquart, Dick Manchester, Naomi McClure-Griffiths, Dave McConnell, Andrew Melatos, Don Melrose, Luciano Nicastro, Ben Stappers, Dan Stinebring, Olaf Skjaeraasen, and Norbert Wex.

\section{References}

Ball, L. et al. 1999, ApJ, 541, L39

Ball, L., Kirk, J. 1999, Astroparticle Physics, In Press

Cominsky, L. et al. 1994, ApJ, 427, 978 
Grove, J. et al. 1995, ApJ, 447, L113

Hirayama, M. et al. 1996, PASJ, 48, 833

Hirayama, M. et al. 1999, ApJ, 521, 718

Johnston, S. et al. 1992a, MNRAS, 255, 401

Johnston, S. et al. 1992b, ApJ, 387, L37

Johnston, S. et al. 1994, MNRAS, 268, 430

Johnston, S. et al. 1996, MNRAS, 279, 1026

Johnston, S. et al. 1999, MNRAS, 302, 277

Kaspi, V. et al. 1995, ApJ, 453, 424

Kirk, J. et al. 1999, Astroparticle Physics, 10, 31

Manchester, R. et al. 1995, ApJ, 445, L137

McClure-Griffiths, N. et al., 1998, ApJ, 492, L49

Melatos, A. et al. 1996, MNRAS, 275, 381

Tavani, M. \& Arons, J. 1997, ApJ, 477, 439

Wex, N. et al., 1998, MNRAS, 298, 997 